\title{
FUTURE COSMIC MICROWAVE AND COSMIC INFRARED
}

\section{BACKGROUND MEASUREMENTS}

JOHN C. MATHER

Code 685, Laboratory for Astronomy and Solar Physics

NASA Goddard Space Flight Center, Greenbelt, MD 20771 $U S A$

\begin{abstract}
.
Cosmic microwave and infrared background radiation (CMBR and CIBR) measurements in the near future have the potential to greatly advance our knowledge of the early universe. New instrument and space technology will soon enable much better measurements of both.
\end{abstract}

\section{Cosmic Microwave Background Anisotropy}

The CMBR anisotropy has been well measured on angular scales of $>7^{\circ}$ by the COBE DMR instrument, and the data from the first two years of observation (Bennett et al. 1994) agree well with the maps from the first year (Smoot et al. 1992, Bennett et al., 1992; Wright et al. 1992). The maps of anisotropy can be most conveniently described in terms of their spherical harmonic representation. A wide variety of measurements at smaller $\left(\sim 1^{\circ}\right)$ angular scales suggest that there really is a detection of excess fluctuations at the "Doppler Peak". This is the name given to a bump in a plot of the $c_{\ell}^{2}$ versus $\ell$, where $\ell$ is the spherical harmonic order and $c_{\ell}^{2}$ is the sum of the squares of the spherical harmonic coefficients of order $\ell$. Doppler did not of course predict this peak, but our Russian colleagues tell us that it was discussed by Sakharov and should properly be called "Sakharov Oscillations".

In theory, these oscillations are the acoustic modes of the primordial material at the time of decoupling. Their scale size is the horizon length at that time, and their amplitude is driven by the density inhomogeneities frozen in from the Big Bang. Therefore, measurement of their detailed properties 
would be extremely revealing, and could help determine the total density $\Omega$, the cosmological constant $\Lambda$, and possibly the properties of the dark matter that govern damping of the acoustic waves.

Not only should there be large scale anisotropy and Sakharov oscillations, but the CMBR may be slightly polarized by them. This is the result of anisotropic radiation becoming polarized by its last Compton scattering.

New technology is now available to make these observations possible. Improved bolometer detectors and microwave amplifiers make better receivers with far better noise levels, bringing raw sensitivities within factors of 2 or 3 of the intrinsic photon noise of the CMBR. Observations from colder and dryer sites are now working well, and several groups are planning or proposing space missions. It now seems possible that the next decade will bring all-sky maps at angular resolutions of $0.5^{\circ}$ and sensitivities limited by the cosmic fluctuations themselves. For smaller angular scales, the same sensitivity can be expected, but full sky coverage will be much more difficult and less important.

Measurements of this accuracy would enable us to test the tensor theories of gravity, which would modify the lowest order terms in the harmonic spectrum. On medium scales, the spectrum can be compared to predictions that $n=1$ precisely, according to the simple versions of inflation. Searches for cosmic strings will also be possible. On smaller scales, the Sakharov oscillations could be detected, yielding the main unknown parameters of the Big Bang and dark matter. On even smaller scales, the Sunyaev-Zeldovitch effect and the peculiar velocities of clusters may be detectable, and with great persistence we may even find the transverse velocities of clusters from the polarization of their scattered light.

\section{Cosmic Microwave Spectrum}

The spectrum of the CMBR has been well measured by the COBE FIRAS instrument (Mather et al. 1994) but the accuracy was limited by systematic error problems. We believe that a factor of 3 improvement is possible using the existing data, but we do not know whether to expect a detection of a spectrum distortion or an upper limit. The energy required to keep the intergalactic medium ionized should also distort the spectrum, so a better measurement will restrict the epoch of reionization.

At wavelengths $>5 \mathrm{~mm}$, the FIRAS measurement is not restrictive, and direct measurements are needed. Two teams are preparing balloon experiments to measure the longer wavelength spectrum, and at least one satellite experiment has been proposed. It will be possible to measure the chemical potential distortion parameter $\mu$ much better than FIRAS could do, and to search for free-free emission from the reheating. 


\section{Cosmic Infrared Background Spectrum}

The CIBR contains the accumulated emissions of generations of stars, galaxies, quasars, and other objects. It is even possible that some of the CIBR was produced before the decoupling, for instance by decay of unstable elementary particles. The present limits on the CIBR are weak, and it could contain an amount of energy comparable to the CMBR. A measurement of the CIBR would provide firm integral constraints on the evolution of most categories of luminous objects, especially galaxies.

The COBE DIRBE team is proceeding well in the analysis of the flight data, and limits on the CIBR are significantly below the foreground emission. The foreground from interplanetary and interstellar dust and starlight has many components and an accurate model requires much thought and analysis. The residuals from the fitting have a spectrum that resembles the foreground, so it is plausible that they represent errors in the foreground models. The calibrated sky maps have been delivered to the public and are available on the Internet.

Indirect measurements or limits have also been obtained by observations of very high energy gamma rays from a quasar. If the CIBR is sufficiently intense, then the gamma rays will be attenuated by pair production of electrons and positrons. Little attenuation is seen (Stecker and DeJager 1993, Slavin and Dwek 1994).

It is unlikely that a large signal-to-noise ratio detection of the CIBR will be possible from the DIRBE data, so more work will be needed. The main foreground source is interplanetary dust, so a space mission to observe outside the cloud would be very helpful. The solar flux falls as $1 / r^{2}$ and the dust density falls roughly as $1 / r$, so it helps a great deal to go even a little farther from the Sun. At least two such missions have been proposed to NASA and one to ESA, so it seems that they are technically feasible. The possibility to fly them will depend on on the level of scientific interest.

\section{References}

Bennett, C. L. et al. , 1992, ApJ, 396, L7

Bennett, C. L., et al. , 1994, ApJ, 436, 423-442

Dwek, E., and Slavin, J., 1994, ApJ, 436, 696-704

Mather, J. C. et al. , 1994, ApJ, 420, 439-444

Smoot, G. F. et al. , 1992, ApJ, 396, L1

Stecker, F.W., \& Dejager, O.C., 1993, ApJ, 415, L71.

Wright, E. L. et al. , 1992, ApJ, 396, L13 